for long periods. He then described the technique of isokinetic sampling at various points in the ducting, so as to determine with reasonable accuracy the dust burden of gases.

A major carbon-black industry is a post-war development, and its installation in the United Kingdom where there are no wide open spaces has led to the development of the elaborate technique described by D. L. H. Allan (Philblack, Ltd.). The manufacture is, of course, a deliberate attempt to produce 'soot'. The soot-laden gases from the burning of a petroleum oil fraction under controlled conditions are taken to electrostatic precipitators, which act essentially as agglomerators, rendering the 'black' capable of arrestment by cyclones. Any residual 'black' in the off-gases is then remgved by water scrubbing followed by wet electrostatic precipitation or by bag filtration. The off-gases still contain enough hydrogen sulphide to be offensive and are disposed of by flaring or burning in a special furnace.

C. J. Stairmand and R. M. Kelsey (Imperial Chemical Industries, Ltd., Billingham Division), taking as typical examples of cyclones the Scroll collector, the high-throughput cyclone, the high-efficiency cyclone and the multicyclone, dealt with the uses to which these can be put as arresters of grit and dust and outlined the procedure for predicting their performance. Cyclones can also serve an extremely useful purpose in reducing the load on more expensive and elaborate secondary collectors; for example, the use of high-efficiency cyclones in connexion with electrostatic precipitators is well known.

Discussion of the various papers related mainly to elaboration of miscellaneous points, and it was noticeable that those papers dealing with visible pollution-cement, grit, carbon black-produced the longer discussions.

\title{
THE EXPERIMENTAL APPROACH TO THINKING
}

$\mathrm{W}^{\mathrm{n}}$ HEN the old Persian philosopher Avicenna, a thousand years ago, felt perplexed and unable to follow a syllogism, he would go to the mosque and pray for his understanding to be opened and his difficulties to be smoothed away. If he were with us now, we could scarcely offer him wiser counsel. The charge made by Graham Wallas a quarter of a century ago that when the text-books of psychology pass from the simpler to the more elusive mental processes "they become in almost every case entirely useless" is almost as pertinent to-day. This is not the place to meditate on the possible causes of this sad state of affairs; but we can feel much sympathy with Sir Frederic Bartlett's aim, in his Friday Evening Discourse given at the Royal Institution, London, on March 4, to use the experimental method in the hope of casting a ray of light on the darkness of our thoughts. He would be content if he could demonstrate that problems of human thinking can fruitfully be made a subject for experiment; but he would not wish to imply that an experimental approach to the study of thinking is new. Four years ago, Prof. G. Humphrey, in a scholarly work ${ }^{1}$, gave an account of a variety of such studies extending over a period of half a century, from the Würzburg School onwards; and in our laboratory at Manchester we have for some time been pursuing the experimental study of thought.

Sir Frederic takes his point of departure from long experience in the investigation of skill and declares that thinking "is in the order of skilled behaviour" in which symbols of various kinds rather than bodily movements are employed. He cites interesting examples from everyday life in which skill in filling a gap is required, such as, for example, when a page is missing from a book we are reading or when, in tracking a target, we lose it for a time and then pick it up again.

He maintains that his analogy between thought and skill is "the most reasonable suggestion and the one most closely in agreement with evidence of a general character". The suggestion, he believes, is also convenient because of recent advances in our knowledge of skills. He does not, however, tell us why it is the most reasonable suggestion, nor does he indicate the general evidence to which he refers.
Sir Frederic observes that bodily skill begins with some outside signal and is "commonly sustained by a succession of such signals". But is not the characteristic feature of thought that it is essentially $a_{0}$ central process, not sustained by outside signals? If so, the analogy between skill and thought may perhaps not be as fruitful as appears at first sight.

All his proposed experiments are of a similar type or form. Evidence or information is presented to the subject with gaps in it, and in such a way "that the gaps must be filled up by a sequence of related steps or moves". The problems vary in complexity and in the number of items of information provided. At one extreme, the subject is given, say, the digits $1,3, \ldots 17$ and asked to fill in the missing items. At the other extreme, a passage is presented describing results of experiments concerned with the effects of temperature on sensitivity, and the subject has to answer certain questions about the conclusions established by the evidence. As examples of intermediate problems we may take as one case where the subject is given the sets $1234: 2134$ : 2143 , and has to continue to change the positions of the numbers until he reaches the final arrangement that seems to be aimed at; another example is where the subject is given sixteen letters of the alphabet set out in random order and has to answer the following questions: If any of these letters is chosen at random, how many questions need I ask in order to discover which it is? What are the questions? Put them in order.

Sir Frederic believes that the underlying experimental pattern is quite general and capable of being applied equally to popular and to scientific systems of thought. He employs the idea of 'directionality' in a sense different both from that of N. R. F. Maier and from the earlier Würzburg idea of 'determining tendency", defining it in terms of "more or less regularly increasing probability of next step". It is not clear, however, how this probability is itself to be measured in the experimental situation. Nor is it clear why Sir Frederic thinks it 'certain' that the storage of information (or memory) does not impose 'directionality' upon it. Do not his own distinguished researches on remembering suggest the contrary? 
The belief, which Sir Frederic seems to approve, that thinking is "tied up with the solution of problems" may be seriously questioned. It does not appear to be necessary for Sir Frederic's proposals and it is a pity that he has not criticized it. The cliché -so often met in the American text-booksthat "thinking is problem-solving" overlooks the fundamental portion of our thinking which has to do with the detection or identification of problems. The very word 'problem' is hard to pin down to any useful meaning, and in much of our thinking we are groping to find out what needs to be done rather than how it needs to be done.

Whenever we consider experiments in psychology, we should see them in perspective and recognize that there are other methods just as important. Without in any way diminishing the value of Sir Frederic's suggestions about thought and skill, we have to bear in mind that most illuminating insights into thinking have come from men gifted with an intuitive understanding of their own mental life, from novelists and poets like Proust and Keats, as well as from men of science and mathematicians like Helmholtz, Poincaré, Graham Wallas and Hadamard. Above all, we are indebted to Freud for a far-reaching conception of thought which is at once biological in character and based on profound clinical observa. tion. The scope of the psychology of thought must cover the developmental sequence of mental growth, pathological thought, as well as comparative studies in different cultures. In these spheres, it is vital to supplement experiment with other methods of inquiry. Sir Frederic's proposed experiments are drawn from his interest in skill. A full programme of experimental inquiry into thinking must be founded upon a deep understanding of the characteristic features of thought during the period of development, of non-conscious thought structures, of the psychopathology of thought and of social and cultural influences on thinking. ЈоHN COHEN

${ }^{1}$ Humphrey, G., "Thinking: An Introduction to its Experimental Psychology" (Methuen, London, 1951).

\title{
A NEW AETHER-DRIFT EXPERIMENT
}

\author{
By DR. L. ESSEN \\ Electricity Division, National Physical Latoratory, Teddington, Middlesex
}

\begin{abstract}
$\mathrm{T}$ HE special theory of relativity is so well established by indirect evidence that the significance of the original æther-drift experiments has decreased, and the experimental discrepancies in the results obtained have caused few misgivings. Such discrepancies should, however, be examined if further developments in experimental technique enable new and possibly more precise methods to be
\end{abstract} employed.

The possibility of performing an experiment similar to that of Michelson and Morley but using short radio waves in place of light waves has been discussed in several letters in Nature ${ }^{1-4}$, and the experiment outlined in one of these ${ }^{2}$ has now been completed at the National Physical Laboratory.

A cylindrical cavity resonator of length $16 \cdot 866 \mathrm{~cm}$. and diameter $8.075 \mathrm{~cm}$. was used to control the frequency of an oscillator at approximately 9,200 Mc./s. The resonator was mounted with its axis horizontal and was rotated continuously in a horizontal plane at a rate of about one turn per minute, the frequency of the oscillator being measured by comparison with a quartz standard at intervals of $45^{\circ}$ during the rotation.

The theory of the method may be described in the following way in order to bring out the points of similarity to the optical method. The cavity resonates when its length is a whole number of half - wavelengths and the resonant frequency is given by :

$$
f=\frac{v}{\lambda}=\frac{n v}{2 l}
$$

where $v$ is the phase velocity, $\lambda$ the wave-length, $l$ the length of the resonator and $n$ the number of half - wave-lengths. In this condition the time taken for the phase of the wave to travel to and fro between the end faces of the resonator is $1 / f$. If now because of the earth's orbital motion a relative velocity $q$ be postulated between the resonator walls and the medium of propagation within it, then just as in the optical case, this time becomes:

$$
t_{1}=\frac{2 l}{n v}\left(1+\frac{q^{2}}{v^{2}}\right)
$$

when the axis of the resonator is in line with the orbital motion, and :

$$
t_{2}=\frac{2 l}{n v}\left(1+\frac{1}{2} \frac{q^{2}}{v^{2}}\right)
$$

when it is at right angles to it. The resonant frequency and therefore the frequency of the controlled oscillator should thus vary by a fractional amount of $q^{2} / 2 v^{2}$ on rotation, there being two minima and two maxima for a complete rotation of $360^{\circ}$.

In the optical experiment, interference fringes are formed between two beams of light which have followed paths at right angles to each other. On rotation of the interferometer through $90^{\circ}$, the positions of the paths are interchanged and the difference in transit time is reversed, giving a fractional change of $q^{2} / v^{2}$ which is observed as a fringe displacement. Another difference from the present experiment is that whereas the phase velocity of the light waves is very nearly equal to the free space value $c$, it is greater than $c$ for the radio waves and depends on the diameter of the cylinder in which the waves are travelling. The calculated frequencychange is accordingly only 3 parts in $10^{\circ}$ for the resonator and frequency employed if the earth's orbital velocity is taken as $30 \mathrm{~km}$./s., while the corresponding fractional time change in the optical method is 1 part in $10^{8}$.

The results obtained are summarized in Table 1, which gives the average of each set of observations reduced to a convenient form by a correction for linear drift and the subtraction of a constant term.

In the last column is given the amplitude of the second harmonic periodic term as determined by harmonic analysis. The figures in the last line are obtained by averaging the observations for every rotation and not by averaging the mean figures given above. The averaging is permissible because the 